\title{
L'AMÉNAGEMENT DES FORCES HYDRAULIQUES DU DANUBE AUTRICHIEN
}

\section{LA CENTRALE DANUBIENNE D'ASCHACH}

\author{
PAR \\ F. MAKOVEC *
}

\section{Généralités.}

Les eaux du Danube qui est, après la Volga, le plus puissant fleuve d'Europe, sourdent du versant est de la Forêt-Noire allemande, à une altitude d'environ $1000 \mathrm{~m}$, et s'ćcoulent par les deux ruisseaux Brege et Brigach donnant naissance au fleuve proprement dit. Long de $2848 \mathrm{~km}$, le fleuve, qui se dirige principalement vers l'est, traverse ou touche huit pays différents: l'Allemagne, l'Autriche, la Tchécoslovaquie, la Hongrie, la Yougoslavie, la Roumanie, la Bulgarie, et l'Union Soviétique. Il débouche enfin dans la mer Noire par un impressionnant delta (fig. 1). Sur son long parcours, le Danube recoit les eaux de nombreux affluents et s'écoule tour à tour par de larges plaines et par d'étroites vallées encaissées. La figure 2 montre le profil en long du fleuve, tout en indiquant la croissance de son débit, depuis la source jusqu'à l'embouchure.

\section{Le Danube, voie de navigation.}

Le caractère du Danube, en tant que ligne de démarcation naturelle bien définie et comme importante voie de communication, a été reconnu dès l'Antiquité. De Ratisbonne jusqu'aux basses plaines roumaines, il représentait la limite septentrionale de l'Empire Romain. Les innombrables campements militaires et forteresses des légions romaines implantés le long de sa rive droite ont donné naissance à nombre de localités et de villes riveraines d'aujourd'hui. Ce fleuve important représentait en toute saison de l'année une voie de navigation et de transport commode pour les bateaux à rames et les radeaux.

A la suite de l'effondrement de l'Empire Romain, l'ensemble de la région danubienne connut les dévas-

* Directeur Technique des Constructions de l’« Oesterreichische Donaukraftwerke, A.G.». tations de l'époque trouble des grandes invasions. Charlemagne qui, il y a plus de mille ans, à la tète de l'armée des Francs, descendit le fleuve jusqu'à Vienne et devint maitre de l'ensemble des territoires en decà de la frontière hongroise, reconnaissait l'importance exceptionnelle du Danube en tant que voie de navigation. Il fut aussi le premier à essayer de relier par un petit canal le Main au Danube, dans le but d'assurer ainsi, sur des bateaux à rames et des radeaux, le transport de soldats, d'armes et de vivres, depuis la vallée du Rhin jusqu'au Danube. Il est intéressant de constater que ce premier projet fut motivé uniquement par des considérations d'économie de guerre, et non de politique commerciale. Les premiers travaux de réalisation du canal (dit fossa carolina) furent effectivement entrepris en l'an 793 après J.-C., mais ils furent abandonnés après la mort de Charlemagne. Quelques vestiges de ce premier canal "Rhin-Main-Danube » sont encore visibles aujourd'hui, au sud de la ville de Nuremberg, notamment sous forme de mares d'eau accumulée et de fosses isolées.

Ce n'est qu'un millénaire plus tard que cette idée de Charlemagne fut enfin réalisée par le roi Louis Ier de Bavière.

De 1836 à 1846, donc en dix ans, fut construit un canal de navigation d'une longueur de $172 \mathrm{~km}$, qui relie Bamberg à Kehlheim. Ce canal a une profondeur de 1,00-1,50 m et une largeur du plan d'eau de $15 \mathrm{~m}$; il est équipé de cent écluses, chacune d'une longueur de $32 \mathrm{~m}$ et d'une largeur de $4,5 \mathrm{~m}$. Les chalands se déplacent au moyen de rames ou sont halés par des chevaux.

Ce canal n'eut malheureusement pas le succès économique escompté, car il fut dépassé par l'évolution impétueuse de la technique alors que sa construction - qui dura dix ans - n'était pas encore achevée. Les trains à vapeur, mis au point à la même époque, étaient nettement plus rapides, 


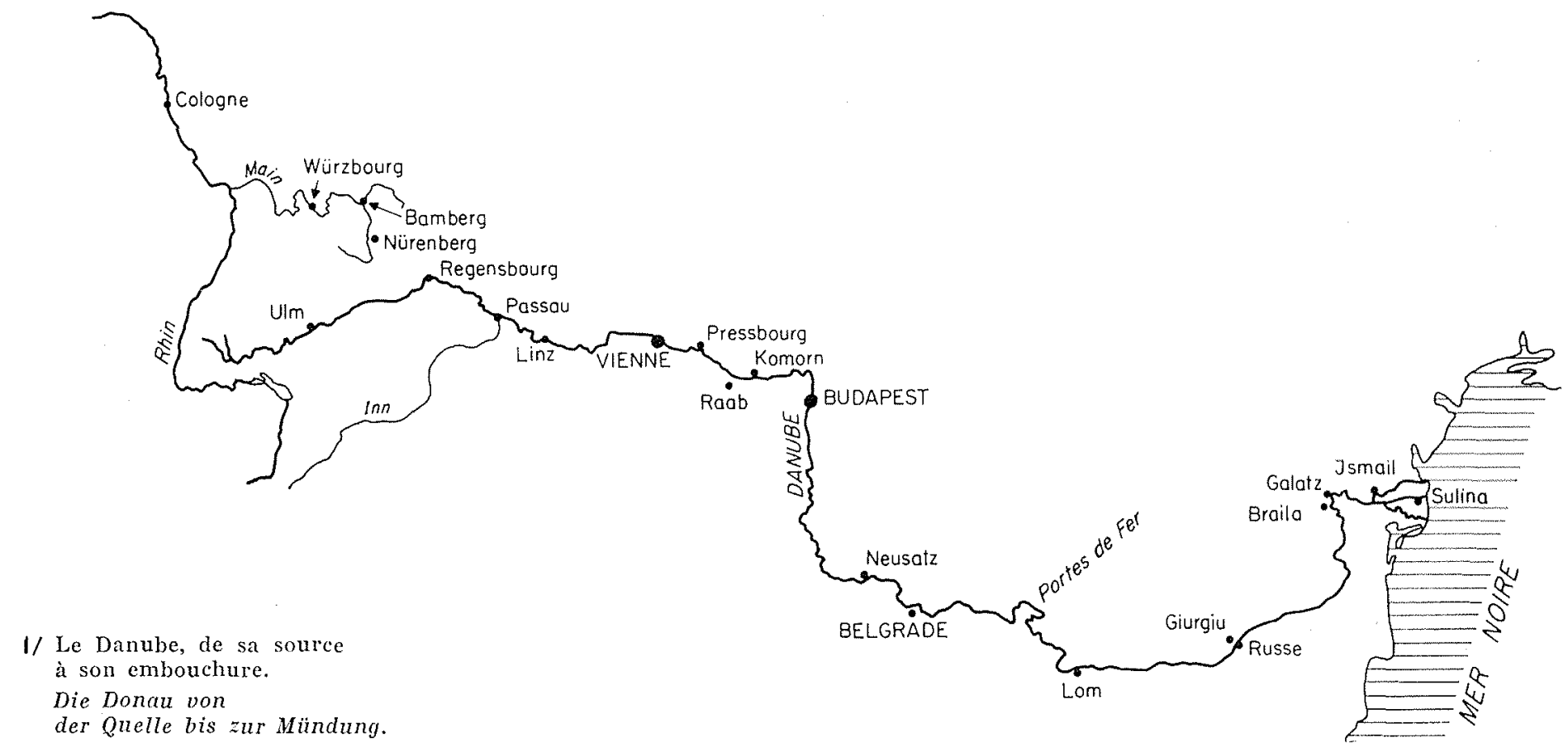

der Quelle bis zur Mündung.

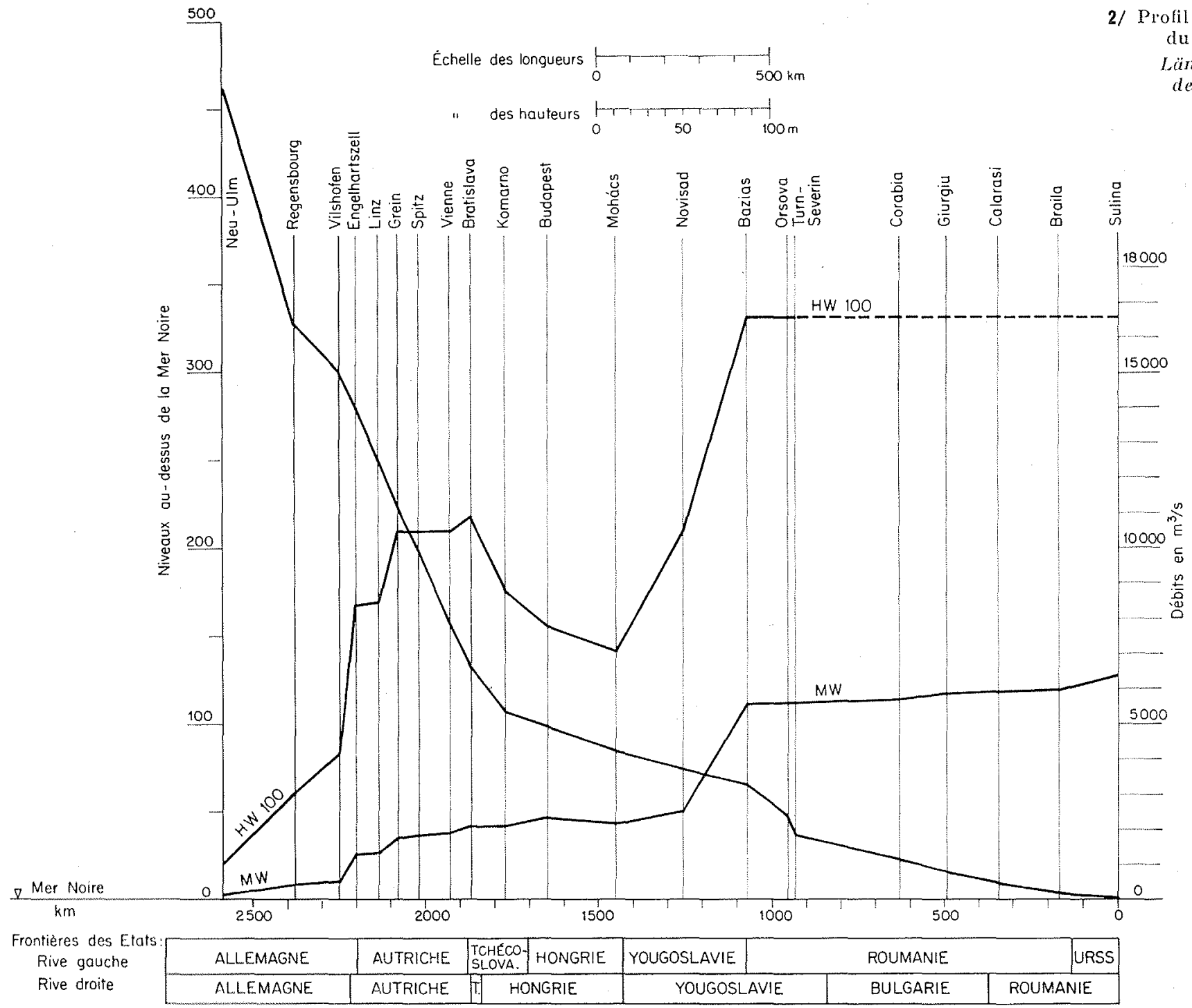


plus puissants, et aussi plus confortables. De plus, les bateaux construits après le début des importantes régularisations fluviales et l'avènement de la navigation à vapeur, étaient tellement grands qu'ils ne pouvaient plus passer par ce canal étroit et peu profond. Néanmoins, le canal fut maintenu en service jusqu'en 1943 et connut une navigation animée de petits bateaux, dont la capacité de chargement allait jusqu'a $100 \mathrm{t}$. Au cours de la Deuxième Guerre, le canal fut endommagé en plusieurs endroits par les bombardements, et il ne fut jamais réparé.

Comme nous venons de le dire, le Danube fut, depuis toujours, une voie de communication et de transport importante, mais aux possibilités restreintes du fait que la navigation devait se limiter à des bateaux à rames et des radeaux relativement petits, et que la navigation ver's l'amont, à contrecourant, était extrèmement difficile. Il y a un siècle, la mise en service des bateaux à vapeur amena alors brusquement un changement fondamental. Les nouveaux bateaux, importants et qui furent équipés de puissantes machines, étaient dès lors capables de transporter sans effort, en amont et en aval, des charges bien plus importantes, et en peu de temps la navigation connut un accroissement considérable. Cependant, le fleuve qui, jusqu'alor's, avait été laissé en son état naturel et comportait encore de nombreux bras, des écueils et des bancs de sable, ne pouvait plus répondre aux exigences accrues de la navigation. C'est ainsi que tous les Etats traversés ou touchés par le Danube furent finalement contraints de soumettre le fleuve, jusqu'à son embouchure, à une régularisation el à des mesures de protection importantes. Dans ce cadre, une attention particulière fut accordée à la protection, contre les crues, des nombreuses agglomérations riveraines, sans cesse en extension.

Ce premier plan de régularisation du Danube eut donc pour but la protection contre les crues, ainsi que l'amélioration et la sécurité de la navigation. La réalisation de ce projet imposant et fort coûteux demanda de longues années d'un travail achamé, mais il fit, au moyen d'épis et de digues basses, de destructions, de dragages et d'ouvrages de protection, de ce cours d'eau incertain et encore à l'état naturel, un lit capable de résister mème aux attaques de crues importantes, et dont la profondeur permettait la navigation des grands bateaux. Vers 1900, la majeure partie des travaux de régularisation était achevée sur tonte la longueur du fleuve. A quelques rares exceptions près, la profondeur étail de $2 \mathrm{~m}$ à l'étiage, sur tout le cours du Danube autrichien. Il est évident qu'il était nécessaire de soumettre le fleuve à une surveillance permanente et de compléter les ouvrages de consolidation.

Les avantages offerts par une voie de navigation sûre, d'une longueur de $2500 \mathrm{~km}$, provoquèrent un accoissement considérable de la navigation, et c'esk ainsi que des milliers de bateaux tracteurs et de chalands appartenant à tous les pays d'Europe naviguèrent sur le Danube, depuis Ratisbonne jusqu'à la mer Noire, et mème au-delà, jusqu'à Istanbul et à la Grèce. L'important développement de la navigation exigea la construction d'un srand nombre d'embarcadères, d'entrepòts et d'installa. tions portuaires comportant tous les engins de chargement et les roies ferrées de connexion nécessaires.

\section{Le Danube, source d'énergie.}

Les descriptions données précédemment montrent que jusqu'à la fin du siècle dernier, le Danube n'eut de l'importance qu'en tant que voie de navigation, et que cette importance fut plus particulièrement encore accrue par l'avènement de la navigalion à vapeur. Simultanément cependant, l'évolution impétueuse de l'industrie eut pour conséquence, au cours de la deuxième moitié du siècle dernier, un accroissement permanent des besoins en énergie. Mais à cette époque l'on ne disposa que des installations à vapeur pour répondre à ces besoins. Peu à peu les spécialistes se toumèrent alors vers les forces hydrauliques qui, jusqu'à cette époque, n'étaient pas encore ou seulement peu exploitées. L'on commença ainsi à construire, sur des petites rivieres à forte pente, des retenues et des turbines qui furent directement accouplées à l'usine dont elles devaient assurer l'alimentation en énergie. Le rendement de ces turbines était encore bien faible. Dans la plupart des cas, la force ainsi obtenue fut transmise, au moyen de roues dentées et de courroies, sur un arbre d'entraînement con. tinu monté dans l'usine. Suivant les besoins, les différentes machines étaient alors raccordées à cet arbre par des cordes ou des courroies. Il est certain que l'exploitation des forces hydrauliques créa une nouvelle source d'énergie, mais l'emplacement de l'usine était tributaire du lieu d'implantation de la centrale hydraulique.

L'exploitation de la force hydraulique connut enfin un essor extrêmement rapide par le développement et la mise au point des turbines hydrauliques et des génératrices, et plus particulièrement encore grâce à la nouvelle possibilité de transporter, sur de grandes distances, à n'importe quelle tension, l'énergie ainsi obtenue (en 1891 fut réalisée la première ligne à grande distance à courant alternatif à haute tension, ligne allant de la centrale de Lauffen sur le Necker jusqu'à Francfort-sur-leMain). De nombreuses centrales hydroélectriques furent alors construites sur les rivières d'une cerlaine importance. Bien entendu, il s'agissait encore de centrales isolées, implantées sur la rivière aux endroits permettant une bonne liaison avec la zone d'ulilisation, une construction économique et qui, pour d'autres raisons encore, paraissaient favorables.

Depuis 1900 , la somme d'énergie incroyablement élevée que renferme le Danube autrichien a donné lieu à de nombreux projets hydroélectriques. Comme il ressort de la figure 2, la pente du Danube autrichien est en moyenne de $0,45 \%$, ce qui représente une pente extraordinairement forte. De même, le débit du Danube est très élevé; au long de son cours, sur $350 \mathrm{~km}$, le débit des eaux moyennes passe de 1300 à $2100 \mathrm{~m} 3 / \mathrm{s}$.

On comprendra done facilement que les spécialistes aient sans cesse essayé de faire profiter leur pays de cette immense et intarissable source d'énergie naturelle. Ils furent d'autant plus persévérants dans leurs efforts qu'une faible partie seulement des besoins en énergie - besoins toujours croissants .... pouvait être assurée par des centrales thermiques utilisant le charbon provenant des mines autrichiennes.

Au cours des cinquante dernières années, de nombreux projets ont été élaborés dans lo but d'utiliser les forces hydrauliques du Danube autri- 
chien. Suivant le stade qu'avait atteint la technique à cette époque, la plupart des projets avaient pour objet des centrales implantées sur des canaux de la région de Vienne qui est la plus grande consommatrice d'énergie de toute l'Autriche. Parmi ces projets, il y en avait d'autres qui prévoyaient non seulement la production d'énergie, mais également, par endiguement ou tranquillisation des courants, la suppression des derniers obstacles ayant résisté à une régularisation et compliquant encore la navigation. Cependant, chacun de ces projets présentait le grand inconvénient de n'avoir été conçu quo compte tenu d'un avantage particulier quelconque, sans qu'il soit tenu compte des projets futurs intéressant les parties de rivière immédiatement en aval et en amont. Enfin, une réalisation effective de ces projets de centrales aurait eu pour conséquence la non-utilisation de plusieurs parties de la rivière et, partant, d'énormes difficultés du point de vue navigation et régularisation $d u$ fleuve, en raison du régime complètement modifié de celui-ci. Connaissant ces faits, les spécialistes furent donc amenés à souhaiter un nouveau plan plus complet, prévoyant l'aménagement de toute la partie autrichienne du Danube, d'une part eu égard à la production d'énergie, et d'autre part pour que tous les autres domaines de l'économie nationale et de la vie sociale de l'Autriche puissent en tirer profit.

Ce fut le Prof. Dr O. Vas qui, en 1929, exprima pour la première fois l'idée générale d'une utilisation totale des eaux du Danube autrichien. En suivant ces idées, on essaya ainsi à plusieurs reprises, au cours des années suivantes, de disposer sur le Danube une chaîne ininterrompue de centrales. Il ressortait de ces essais un coefficient d'utilisation de la chute brute du Danube qui variait suivant le nombre de centrales choisi. Bien entendu, il est extrêmement difficile de transformer en une chaîne ininterrompue de centrales l'un des fleuves les plus importants d'Europe, un fleuve qui traverse de larges plaines fertiles et des régions où la population est très dense. Il faut, ici, non seulement tenir compte, dans une large mesure, de tous les aménagements et installations existantes, mais i] faut également se montrer prévoyant et prendre en considération tout ce que peut apporter, dans un lointain avenir, l'évolution future.

L'année 1946 apporta enfin un changement fondamental, en ce sens qu'à l'instigation du Prof. Dr O. Vas, fut fondé un «Comité spécial du Danube» qui, en 1947, chargea la société «Oesterreichische Donauwerke A.G. » de l'élaboration du projet d'une chaîne ininterrompue de centrales sur. le Danube.

\section{Le plan cadre du Danube autrichien.}

Le plan cadre du Danube autrichien - plan avant tout conçu dans l'intérêt de l'industrie électrique - constitue la base de la transformation future du Danúbe en une voie d'eau servant à la fois à la navigation et à la production hydroélectrique. Lieu d'implantation, hauteur de retenue et puissance de chacune de ces centrales seront choisis tels qu'ils permettent une utilisation totale des forces hydrauliques naturellement disponibles. Il est alors nécessaire d'évaluer, suivant leur degré d'importance et d'utilité, le grand nombre de désirs et d'exigences émis par les différents domaines de l'économie publique et de la vie sociale, et d'en tenir compte par la suite. Nous donnerons ci-après une brève description des considérations jouant un rôle décisif dans l'élaboration du projet.

Dans la plupart des cas, il convient de déterminer, en premier lieu, l'endrait où sera implanté un étage de retenue. L'étude d'une carte du cours d'eau et du profil en long correspondant suffit pour obtenir, par la succession d'étroiles vallées et de larges plaines, par la situation des agglomérations les plus importantes et des deltas, un nombre suffisant d'indications relatives aux lieux d'implantation convenables et aux hauteurs de retenue admissibles.

Les vallées étroites à pentes raides, généralement pauvres en agglomérations, permettent normalement de construire une retenue élevée, à des frais acceptables. Dans la plupart des cas, la roche solide se trouve à une faible protondeur, d'où la possibilité d'une fondation simple et sûre. Cependant, en raison de la largeur restreinte de la vallée, la réalisation de la construction est généralement difficile et demande beaucoup de temps.

Les plaines choisies pour la construction d'un étage de retenue offrent le plus souvent suffisamment de place pour que l'on puisse y aménager toutes les installations du chantier. Il est judicieux d'implanter le nouvel ouvrage dans la zone riveraine d'un coude de rivière, à l'écart du fleuve. Ceci nécessite évidemment l'endiguement du coude, mais l'on obtient ainsi également la possibilité de ne prévoir qu'une seule fouille pour l'ensemble de l'ouvrage, et de réaliser celui-ci en une seule fois, sans être gêné par le fleuve et la navigation. Grâce aux engins de terrassement modernes, d'une puissance exceptionnelle, les masses de terre provenant des fouilles peuvent facilement être déplacées et utilisées par la suite pour la construction des longs et hauts barrages de retenue. Lors du choix du site, il faut plus particulièrement tenir compte des agglomérations et villes situées sur le fleuve. Lorsque le nouveau barrage se trouve loin en aval de l'agglomération, celle-ci n'est que peu exposée par la retenue. $\mathrm{Si}$, au contraire, le barrage est très proche, l'agglomération doit être soustraite, au moyen de digues longitudinales et de colmatages, aux effets d'une retenue élevée. Il sera alors nécessaire de prévoir des installations de pompage assurant l'abaissement $d u$ niveau de la nappe souterraine et l'évacuation des eaux d'égouts. Dans le but d'éviter ces complications permanentes et coûteuses, l'on tendra donc à implanter le barrage en amont de l'agglomération. De même, en vue de réduire le prix de la construction et de l'entretien, l'on s'efforcera de n'aménager que des étages de retenue, peu nombreux, mais ayant une grande chute et une importante retenue. Des parties de fleuve sans retenue sont à éviter ou bien à supprimer en approfondissant artificiellement le fond de la rivière, à l'extrémité de la retenue.

La réalisation d'une chaîne ininterrompue de centrales permet l'application de l'accumulation continue. Grâce à cette forme d'exploitation particulière, il est possible, en faisant alterner l'écoulement et l'accumulation, d'augmenter ou de diminuer la puissance de cette chaîne de centrales et de l'adapter ainsi aux fluctuations journalières de la consommation d'énergie, à condition évidemment que les étages de retenue soient équipés, aux deux extrémités de la chaîne de centrales, de bassins d'accumulation suffisamment grands. 
Quant à la navigation, elle exige la suppression des obstacles par surélévation du niveau, et la garantie que les bateaux, sous pleine charge, puissent naviguer, de jour comme de nuit, en double file, même pour un régime minimal du Danube. Suivant les recommandations faites par la Commission Internationale du Danube dont la République d'Autriche est membre, le chenal navigable du Danube serait à approfondir jusqu'à présenter une profondeur d'au moins $2,50 \mathrm{~m}$ à l'étiage. Cette condition pourra facilement être remplie lors de l'aménagement d'une chaine de centrales.

La figure 3 montre de larges plaines plates situées le long du fleuve et qui, lors de crues catastrophiques, se trouvent inondées sur une hauteur de plusieurs mètres. Cette accumulation latérale, retenant plusieurs milliards de mètres cubes d'eau de crues, a pour effet un amortissement des ondes de crue, et elle est notamment d'une très grande importance pour la protection contre les crues de la ville de Vienne. La suppression de ces zones de rétention, par la construction de longues digues de. protection contre les crues, aurait pour conséquence un accroissement des ondes de crue et des débits et, de ce fait, du danger de crue pour toutes les agglomérations situées à proximité du fleuve. Il est donc nécessaire de conserver autant que possible les zones de rétention existantes. Lors du choix du lieu d'implantation et de la hauteur de la retenue, de même qu'au cours de la construction des digues de retenue, l'on veillera donc à ce que les eaux de crue puissent s'écouler librement du fleuve dans les zones de rétention.

Les terres soumises à l'exploitation agricole et forestière devront être conservées, du moins dans la mesure où une telle conservation est possible et économiquement justifiable. Les terres menacées par la retenue seront, ou bien surélevées, ou encore protégées de manière permanente par des digues et des installations de pompage. En ce qui concerne la pêche, la réalisation d'une retenue de rivière apporte, dans la plupart des cas, des avantages. Evidemment, il est possible qu'il soit nécessaire de remplacer par d'autres les différentes catégories de poissons, mais ceci peut être obtenu progressivement par un alevinage s'échelonnant sur plusieurs années.

Il est évident qu'en établissant le projet, l'on devra également tenir compte d'autres demandes et d'autres exigences telles que : amélioration du réseau routier par construction de routes et de ponts, développement des services des Sports et de la Santé, prise en considération des problèmes de la conservation des sites et des monuments, etc.

La figure 3 montre le plan de situation de la chaìne de centrales; la figure 4 , le plan des étages

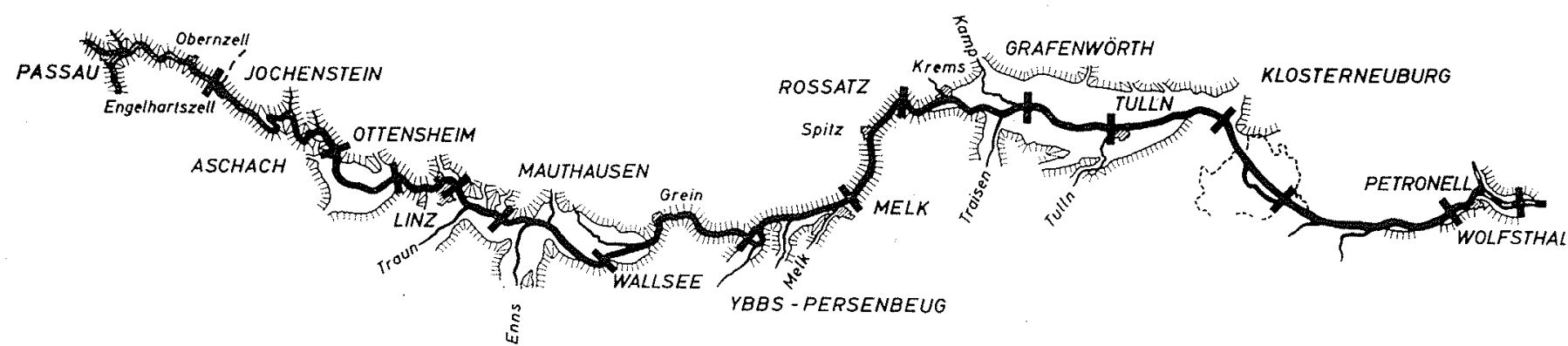

3/ La chaine de centrales du Danube autrichien.

Die Kraftwerkskette der österreichischen Donau.

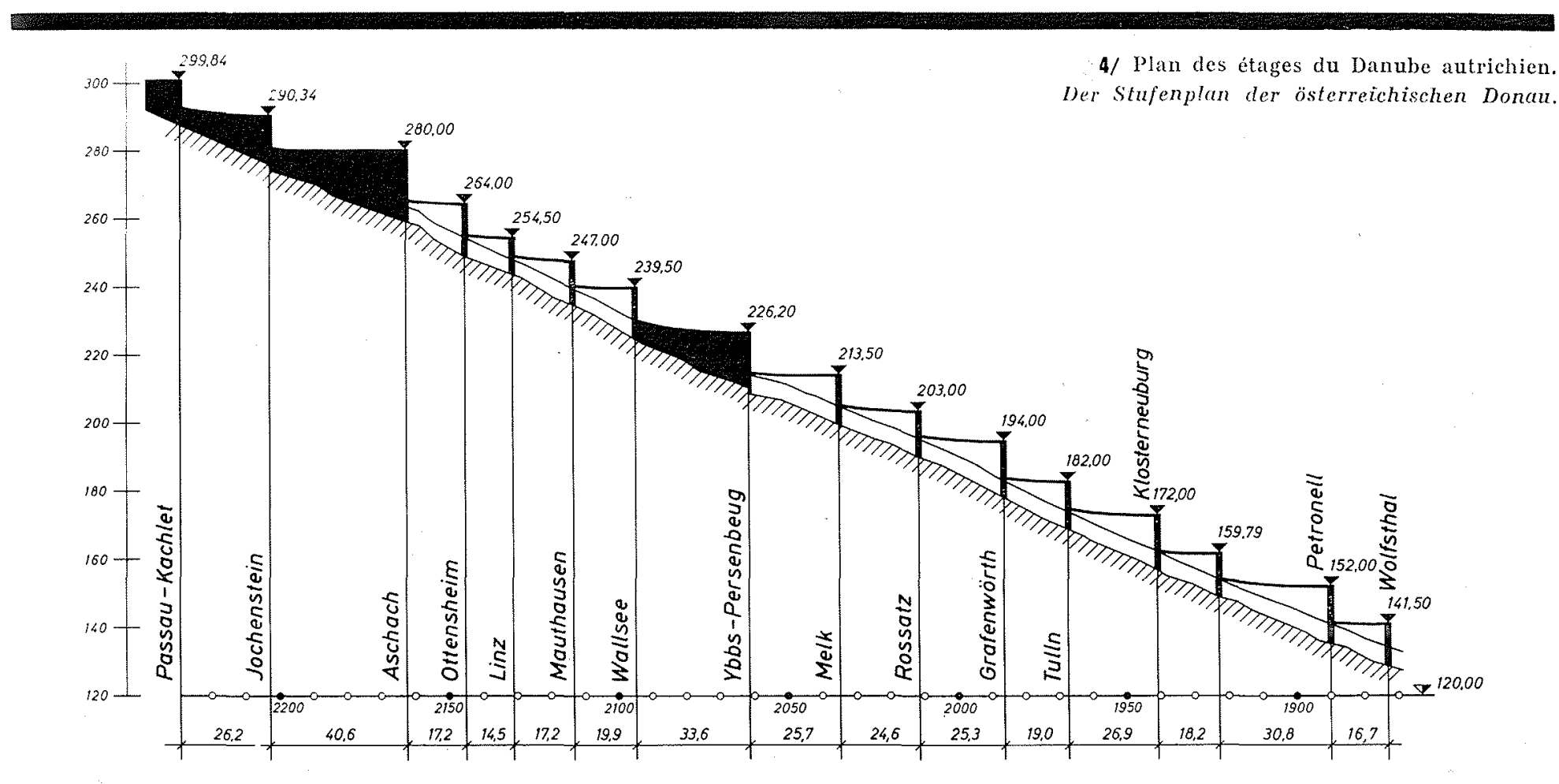




\section{F. MAKOVEC}

\begin{tabular}{|c|c|c|c|c|c|c|}
\hline USINE: & $\begin{array}{l}\text { EMpra- } \\
\text { tEMENT } \\
\text { AU lim }\end{array}$ & 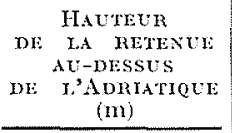 & $\begin{array}{l}\text { HAVTFUR } \\
\text { DE CHVTE: } \\
\text { PAR XIW } \\
\text { (III) } \\
\end{array}$ & $\begin{array}{c}\text { Itvmunes } \\
\text { nimit } \\
(\mathrm{m} / \mathrm{s}) \\
\end{array}$ & $\begin{array}{c}\text { PUISSAYCE * } \\
\text { (MW) }\end{array}$ & $\begin{array}{l}\text { Pronverros } \\
\text { AxNokexis } \\
\text { (GWh) }\end{array}$ \\
\hline Passau-Kachlet. . . . . & 2229,50 & 299,84 & - & $\ldots$ & $\ldots$ & $\ldots$ \\
\hline Jochenstein. . . ..... & 2203,33 & 290,34 & 10,15 & 1750 & 140 & 940 \\
\hline Aschach. . . . . . . . . & 2162,67 & 280,00 & 15,13 & 2040 & 282 & 1587 \\
\hline Ottensheim. . ...... & 2146,15 & 264,00 & 8,44 & 2040 & 142 & 874 \\
\hline Linz. . . . . . . . . & 2131,00 & 254,50 & 5,68 & 1750 & 80 & 507 \\
\hline Nauthausen. . . . . . & 2113,85 & 247,00 & 6,17 & 1800 & 90 & 574 \\
\hline Wallsee. . . . . . . & 2094,24 & 239,50 & 9,19 & 2400 & 200 & 1225 \\
\hline Ybbs-Persenbeug. . . . . & 2060,42 & 226,20 & 11,03 & 2100 & 189 & 1252 \\
\hline Melk. . . . . . . . . & 2034,75 & 213,50 & 8,12 & 2150 & 137 & 907 \\
\hline Rossatz. . . . . . . & 2010,20 & 203,00 & 7,47 & 2150 & 126 & 838 \\
\hline Grafenwörth. . . . . . & 1984,90 & 194,00 & 10,72 & 2150 & 182 & 1203 \\
\hline Tulln. . . . . . & 1965,90 & 182,00 & 8,14 & 2250 & 143 & 952 \\
\hline Klosterneuburg. . . . & 1939,64 & 172,00 & 10,20 & 2640 & 209 & 1300 \\
\hline Wien. . . ......... & 1920,80 & 159,79 & 6,90 & 2640 & 130 & 800 \\
\hline Petronell. . . . . . . . . & 1890,00 & 152,00 & 10,40 & 2300 & 193 & 1280 \\
\hline Wolfsthal. . . . . . . . & 1873,00 & 141,00 & 10,00 & 2400 & 192 & 1330 \\
\hline Total. . . . . . & & & & & $2260^{\star \star}$ & $14434^{* *}$ \\
\hline $\begin{array}{l}\text { * Compte tenu de l'e } \\
\text { * Les centrales de Jo } \\
\text { production. }\end{array}$ & re & les & s en & it & lia & (ie leur \\
\hline
\end{tabular}

5/ Tableau des caractéristiques des centrales.

Tabelle der characteristischen Werte der Kraftuerlie.

6/ La centrale d'Ybbs-Persenbeug (vue aérienne).

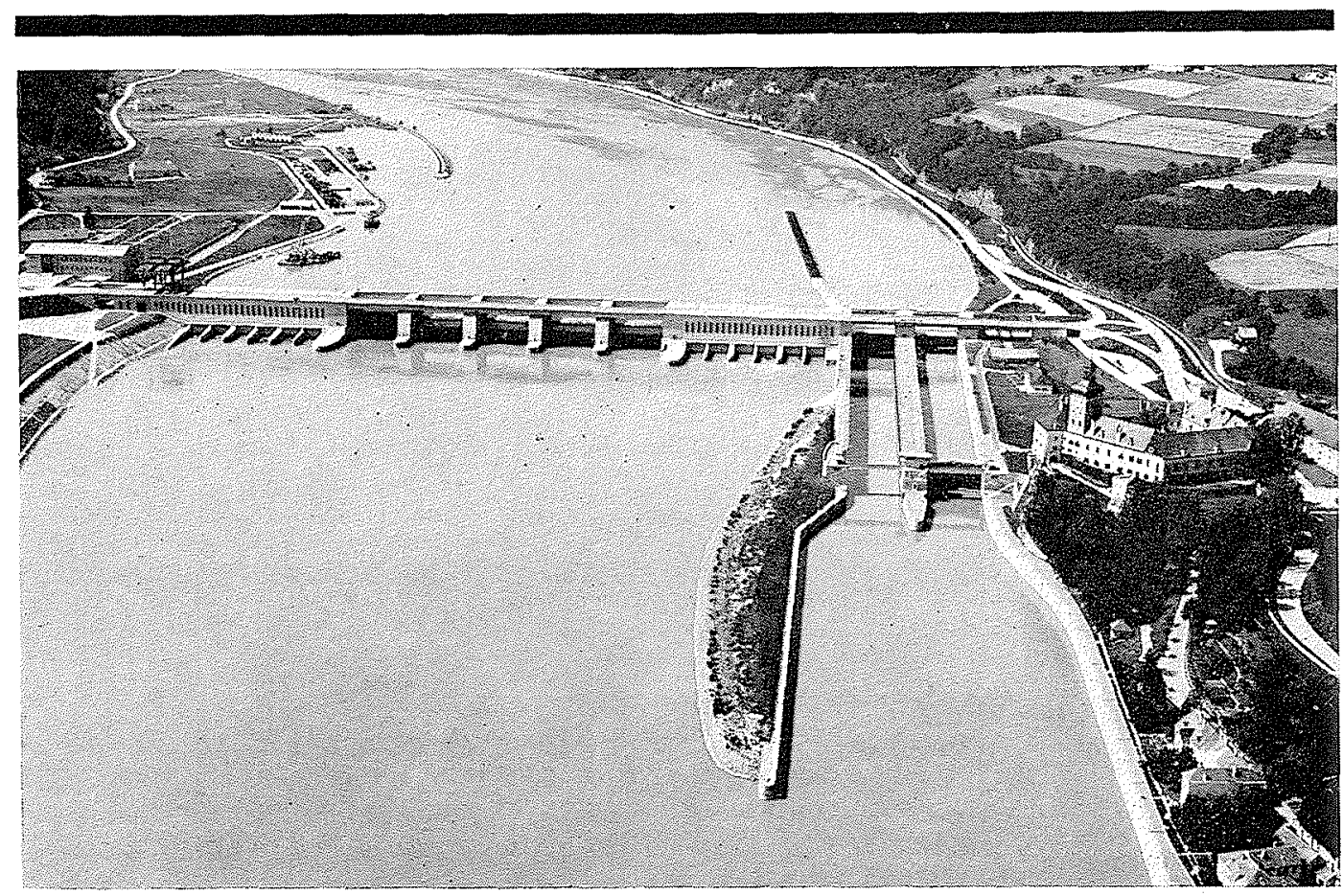

y correspondant, et le tableau de la figure 5 indique les valeurs caractéristiques des différents étages de retenue. Le projet de la Dokw comporte, sur le Danube autrichien, une chaìne ininterrompue de 13 centrales.

Rejoignant à l'ouest la centrale de Jochenstein qui fut achevée en 1956 , et qui est exploitée en commun par l'Allemagne et l'Autriche, la chaîne sera prolongée à l'est par une centrale implantée près de Wolfstal, et qui sera exploitée en commun par l'Autriche et la Tchécoslovaquie.

Comme il ressort du tableau domé dans lat figure 5, I'Autriche disposera, après aménagement de toute la chaîne de centrales, d'une quantité d'énergie annuelle de près de 14,5 milliards de
kWh, dont $40 \%$ en hiver et $60 \%$ en été. La chute brute naturelle du Danube, qui est de $160 \mathrm{~m}$ entre Passau el Wolfstal, sera utilisée à $86 \%$.

Sur les 13 centrales entièrement autrichiennes implantées dans le Danube, la centrale d'YbbsPersenbeug, construite par la Dokw, fut achevéc et mise en exploitation en 1958 (fig. 6). Equipée de machines d'une puissance maximale d'environ $200000 \mathrm{~kW}$, la centrale fournit au réseau autrichien 1250 GWh par an. Au cours de la même année, débuta également la construction de la centrale suivante, qui sera la plus importante. Le stade d'avancement actuel de cette centrale est tel qu'il permettra sa mise en exploitation vers le milieu de l'année 1963. 


\section{Economie de l'énergie électrique.}

En Autriche comme ailleurs, le développement économique apres la deuxieme guerre mondiale a conduit à une forte augmentation des besoins en énergie électrique. En 1961 , la production d'énergie intérieure était de près de 14 milliards de kWh, dont $75 \%$ fournis par des centrales hydroélectriques et $25 \%$ par des centrales thermiques. L'augmentation moyenne annuelle de la consommation est d'environ $7 \%$, et l'on prévoit, pour les dix années à venir, une consommation qui sera le double de ce qu'elle est actuellement, et qui deva etre assurée par la construction d'autres centrales hydroélectriques et thermiques.

Les sources d'énergie intérieures dont dispose l'Autriche se composent de gaz naturel, de pétrole et de lignite, et, avant tout, de forces hydrauliques. Le potentiel hydroélectrique se prêtant à un aménagement, s'élève à 43 milliards de $\mathrm{kWh} / \mathrm{an}$, dont un quart seulement est exploité. De par ses forces hydrauliques, l'Autriche dispose done d'une réserve d'énerge encore inutilisée et intarissable de près de 30 milliards de $k$ Wh, et dont près de la moitié est contenue dans les forces hydrauliques du Danube autrichien.

Comme partout, le côté financier soulève certaines difficultés, car l'aménagement de nouvelles centrales hydroélectriques et de lignes de transmission à longue distance exige une somme annuelle d'au moins 1500 à 2000 millions de sehillings.

\section{LA CENTRALE DANUBIENNE D'ASCHACH}

L'établissement du projet de la centrale d'Aschach, étape de construction succédant à celle de la centrale d'Ybbs-Persenbeug, fut entrepris alors que cette dernière était encore en voie de réalisation. Lo facteur déterminant fut ici, d'une part, le but encore lointain de l'aménagement progressif, sur le cours supérieur du Danube, d'une chaìne de centrales se plaçant entre les centrales de Jochenstein et d'Ybbs-Persenbeug déjà existantes, et de construire en aval et en amont les centrales suivantes, et, d'autre part, le désir de pouvoir disposer de la centrale fluviale la plus importante d'Autriche et d'Europe Centrale, et d'obtenir ainsi, pour le réseau autrichien, un des plus puissants moyens de production d'énergie.

Le présent projet représente le fruit de plusieurs années d'études et de comparaisons de multiples alternatives où il fut non seulement tenu comple des intérêts de la production d'énergie, mais également de toutes les demandes formulées par l'économie et la vie sociale. C'est ainsi que, en accord avec les autorités et les organismes intéressés, toutes les exigences et possibilités de développement furent étudiées et évaluées suivant leur importance et leur intérêt public.

L'idée qui, pendant plus de cinquante ans, a captivé et stimulé les ingénieurs et les spécialistes de l'énergie, se trouve maintenant concrétisée. Le premier projet d'une centrale danubienne dans la région d'Aschach est dû̀ à l'ingénieur FischerRainau qui, en 1910, proposa une centrale en canal, sans barrage. Depus lors, d'autres études et projets suivirent, mais ce n'est qu'en 1959 que la Dokw donna finalement son accord pour la construction du projet décrit ci-après.

La retenue de la nouvelle centrale sur le Danube se trouve au $\mathrm{km} 2162,670$, à environ $2 \mathrm{~km}$ en amont de la vieille ville d'Aschach qui, cependant, ne sera pas affectée par la construction de la centrale. l'ouvrage principal sera implanté en ce point du fleuve et il comportera le barrage, la centrale, l'écluse double ainsi que les bâtiments de service et ceux renfermant les installations de commande. Le fleuve sera surélevé de près de $16 \mathrm{~m}$, jusqu'à la cote 280,50 au-dessus de l'Adriatique. L'accumulation s'étendra sur près de $40 \mathrm{~km}$ vers l'amont, jusqu’à la centrale de Jochenstein située sur la frontière (fig. 7).

Dans la zone de retenue, le fleuve passe par la vallée étroite percée à travers la montagne granitique de la haute Autriche, et il est caractérisé, dans cette région, par des coudes brusques, par des pentes boisées s'élevant jusqu'à une hauteur de $200 \mathrm{~m}$, et par des plaines riveraines relativement étroites, ne donnant place qu'à peu d'agglomérations et de routes. L'aménagement et la construction de celte centrale, à retenue relativement élevée, se trouvent simultanément favorisés et compliqués par ces caractéristiques. L'endroit semble naturellement prédisposé à la retenue, car nulle part ailleurs le cours du fleuve est suffisamment droil, et la vallée assez large, pour pouvoir recevoir l'écluse - d'une longueur d'environ $1 \mathrm{~km}$ dans le sens de l'écoulement - et, transversalement au fleuve, la centrale et le barrage.

L'ouvrage principal, situé au point de retenue, se compose de trois parties :

- barrage à cind passes, sur la rive gauche;

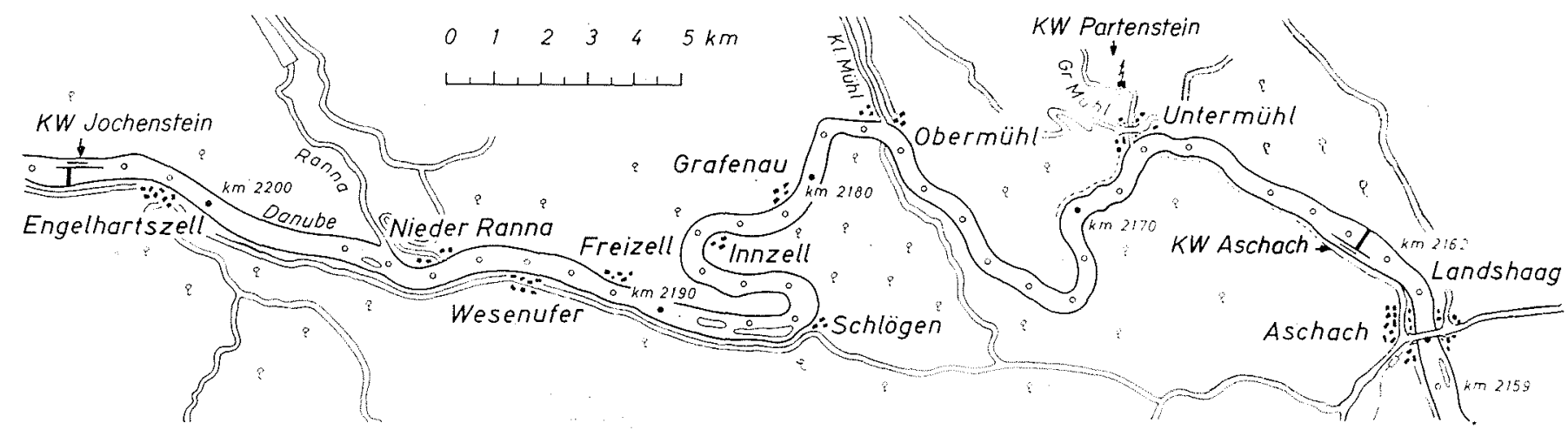

7/ Zone de retenue de la centrale d'Aschach.

Uebersichtiarte des Stauraumes des Kraftwerkes Aschach. 


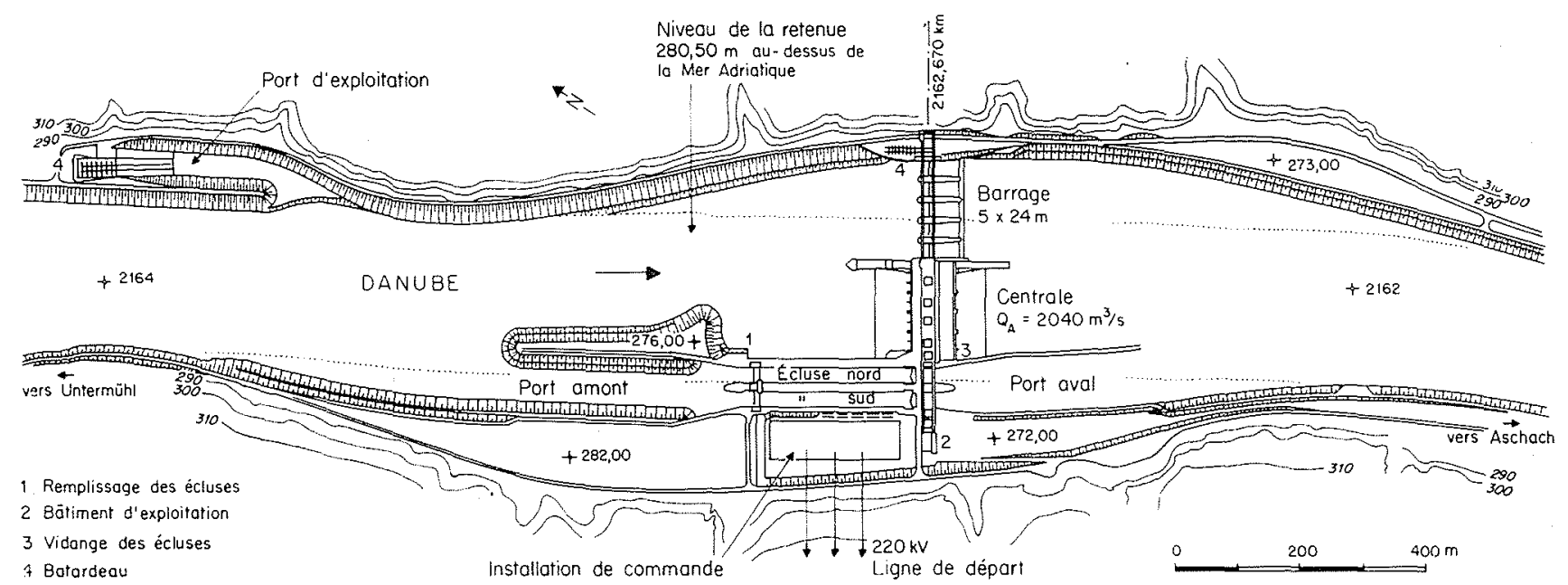

8/ Plan de situation de l'ouvrage principal à l'emplacement de la chute.

Lageplan des Hanptbauwerkes an der Stanstelle.

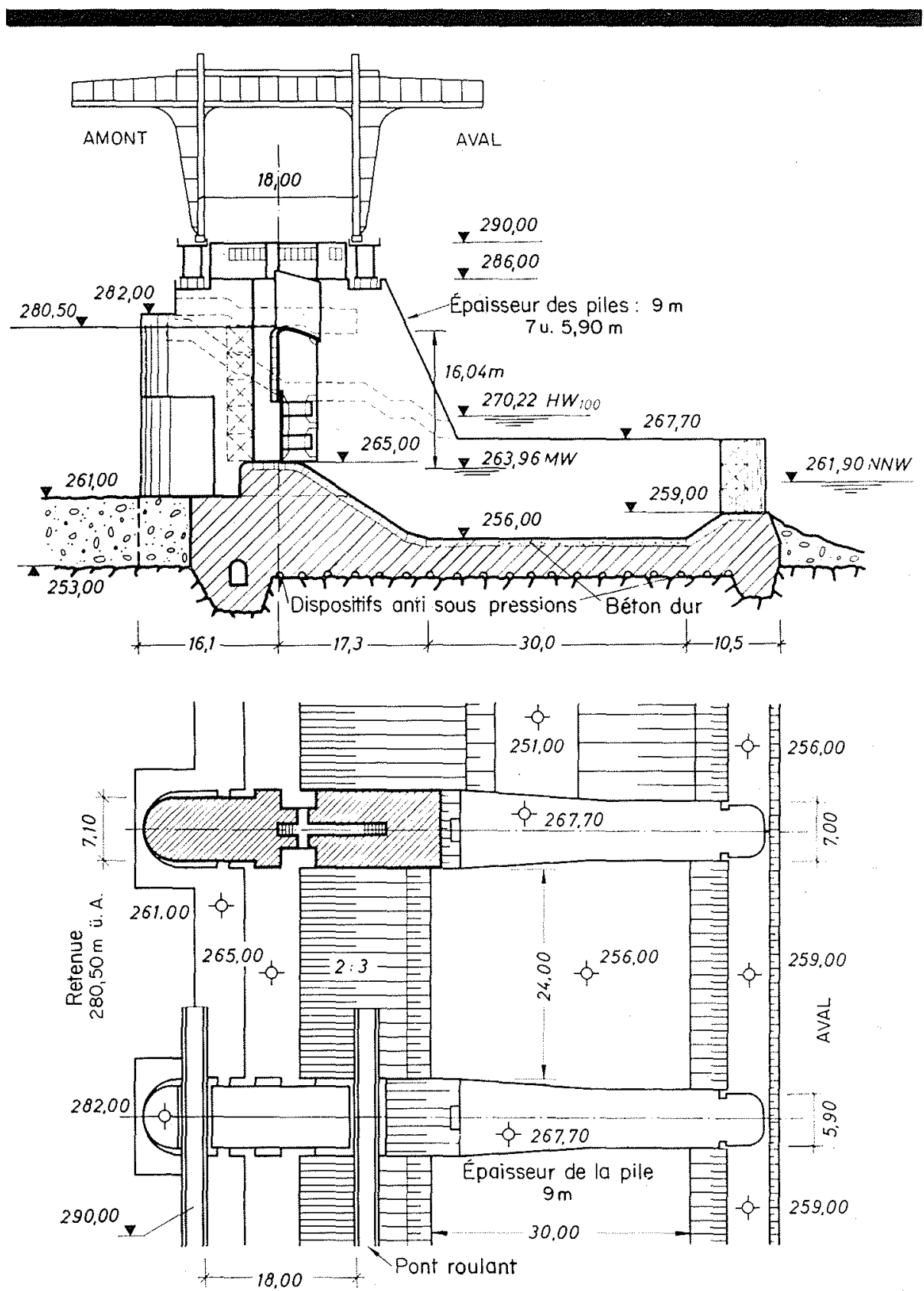

9/ Le barrage.

Wehranlage. 
- une centrale à quatre groupes, au milieu du fleuve environ;

- l'écluse, sur la rive droite.

Le barrage (fig. 9), d'une longueur de $156 \mathrm{~m}$, se compose de cinq passes, chacune ayant une largeur d'éconlement de $24 \mathrm{~m}$, et de quatre piles d'une épaisseur de $9 \mathrm{~m}$. Des vannes à crochet à double porte, d'une hauteur de $15,80 \mathrm{~m}$, servent à la fermeture des pertuis et à la régularisation de l'évacuation des crues. La conception du barrage et, plus particulièrement, la forme et la longueur du bassin de dissipation ont été établies d'après les résultats obtenus par des essais approfondis sur modèle - essais effectués au Laboratoire hydraulique - et qui tenaient également compte des crues exceptionnelles catastrophiques pouvant atteindre $11000 \mathrm{~m}^{3} / \mathrm{s}$, et de forts charriages de glace. Deux de ces passes sont spécialement conçues pour la dérive des glaces et comportent un bassin de dissipation plus profond et renforcé. Ie fond des pertuis ainsi que les parois des piles, sont protégés par des blocs de granite et du béton, contre l'usure due au matérian charrié et aux glaces. De plus, les parties particulièrement exposées sont revêtues d'une armature en acier.

La centrale (fig. 10), qui comporte quatre groupes, sera construite à mi-hauteur, forme de construc tion qui a déjà fait ses preuves pour la centrale d'Ybbs-Persenbeug. Le plafond du batiment des machines se trouve à environ $10 \mathrm{~m}$ au-dessus du niveau de retenue. La centrale est divisée en quatre bâtiments principaux, chacun de ces bâtiments ayant une largeur de $32 \mathrm{~m}$. La centrale comporte quatre turbines Kaplan, d'un diamètre de $8,40 \mathrm{~m}$ chacune. Elles sont disposées de telle manière que, alternativement, leur sens de rotation va vers la gauche ou vers la droite, ce qui permet de grouper en un seul poste de commande, pour deux groupes respectivement, tous les dispositifs de commande mécaniques et électriques, dispositif très avantageux du point de vue exploitation et économie. Chaque groupe est relié à son propre transformateur par des connexions en bloc. Le courant du générateur $(10,5 \mathrm{kV})$ est amené par des barres courtes aux transformateurs, où il est transformé en $220 \mathrm{kV}$. Au moyen d'un câble à huile monophasé, il est alors conduit à l'installation de distribution à l'air libre, située sur la rive droite. Les quatre transformateurs sont montés sur plateforme, à l'air libre, en aval de l'usine. L'usine, d'une longueur

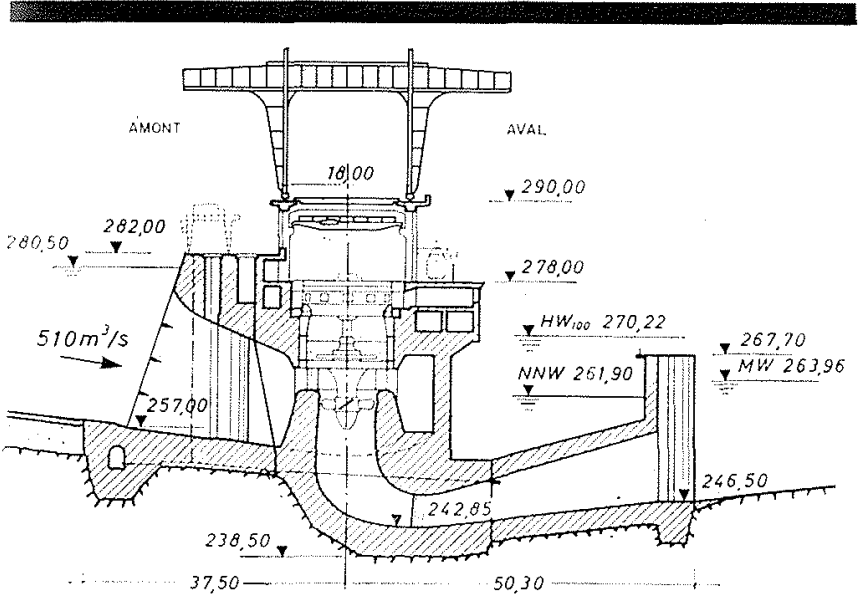

10/ Coupe en travers de la centrale.

Querschitt durch das Kraftwerk. de $150 \mathrm{~m}$ environ, est très bien éclairée des deux côtés; elle est munie de deux ponts roulants intérieurs, d'une charge utile de $16 \mathrm{t}$, indispensables pour le montage et la commande.

Le débit des turbines, soit $2040 \mathrm{~m}^{3} / \mathrm{s}$, correspond au régime du fleuve pendant une période de 60 jours, pour une année moyenne. La puissance de la centrale est de $282000 \mathrm{~kW}$, et la production annuelle possible, de 1680 millions de $\mathrm{kWh}$, pour une année normale. Le bassin versant du Danube, près d'Aschach, couvre $78192 \mathrm{~km}^{2}$. Des $44,8 \mathrm{mil}-$ liards de $\mathrm{m}^{3}$ représentant le débit annuel moyen, 42,4 milliards de $\mathrm{m}^{3}$, soit $95 \%$, passent par les turbines; du point de vue économique de l'énergie électrique, le coefficient d'exploitation est donc exceptionnellement élevé. D'autre part, en raison de sa hauteur de chute utile et de sa capacité d'emmagasinement élevée, la centrale d'Aschach est désignée pour être la centrale pilote de l'accumulation continue envisagée pour la future chaine de centrales entre Aschach et Ybbs-Persenbeug. Grâce à cette accumulation continue, il sera possible d'adapter, dans certaines limites, la production d'énergie aux variations journalières des besoins en énergie.

La pile de séparation spéciale, aménagée entre la centrale et le barrage, remplit des fonctions hydrauliques et constructives, et renferme deux petits groupes destinés à assurer les besoins propres en énergie de tout l'ouvrage principal.

Les dimensions de l'écluse sur la rive droite (fig. 11,12 et 13) correspondent aux normes appliquées aux autres écluses sur le Danube, c'est-àdire celles de Passau, de Jochenstein et d'YbbsPersenbeug. Les deux chambres d'écluse ont chacune une largeur de $24 \mathrm{~m}$ et une longueur utile de $230 \mathrm{~m}$; chacune de ces chambres peut recevoir un train de touage complet constitué d'un remorqueur et de quatre chalands jumelés, ayant chacun une charge utile de $1200 \mathrm{t}$.

Suivant le niveau aval respectif, le remplissage et la vidange d'une chambre d'écluse exigent de 90000 à $120000 \mathrm{~m}^{3}$ d'eau. Le remplissage et la vidange ne devront pas excéder 15 minutes, afin d'éviter aux bateaux une attente inutile; de plus, les chambres d'écluse, ainsi que les avant-ports en amont et aval, devront ètre exempts de tout phéno. mène de hausse ou d'abaissement, et de tout autre courant perturbateur. Alors que pour le remplissage des écluses des centrales de Jochenstein et d'Ybbs-Persenbeug, qui exigent une quantité d'eau nettement inférieure, il est encore possible d'amener l'eau du port amont et de l'évacuer vers le port aval, ce procédé aurait conduit, pour la centrale d'Aschach, à des difficultés énormes. En effet, si l'on respectait la durée maximale de $15 \mathrm{mn}$ fixée pour le remplissage et la vidange, ceci entraînerait des phénomènes considérables de flots et de rabattements du plan d'eau, qui mettraient fortement en danger les trains de chalands entrant et sortant. Afin d'éviter ces difficultés et dangers, l'eau de remplissage est amenée directement de la large retenue que forme le fleuve, au moyen d'un ouvrage de remplissage spécial aménagé à la tête amont de l'écluse. Lors de la vidange des écluses, elle est évacuée en aval de l'écluse par un étroit ouvrage de vidange situé entre l'usine et la tête aval de l'écluse.

De plus, étant donné la profondeur de la surface rocheuse, il est extrèmement facile de construire 

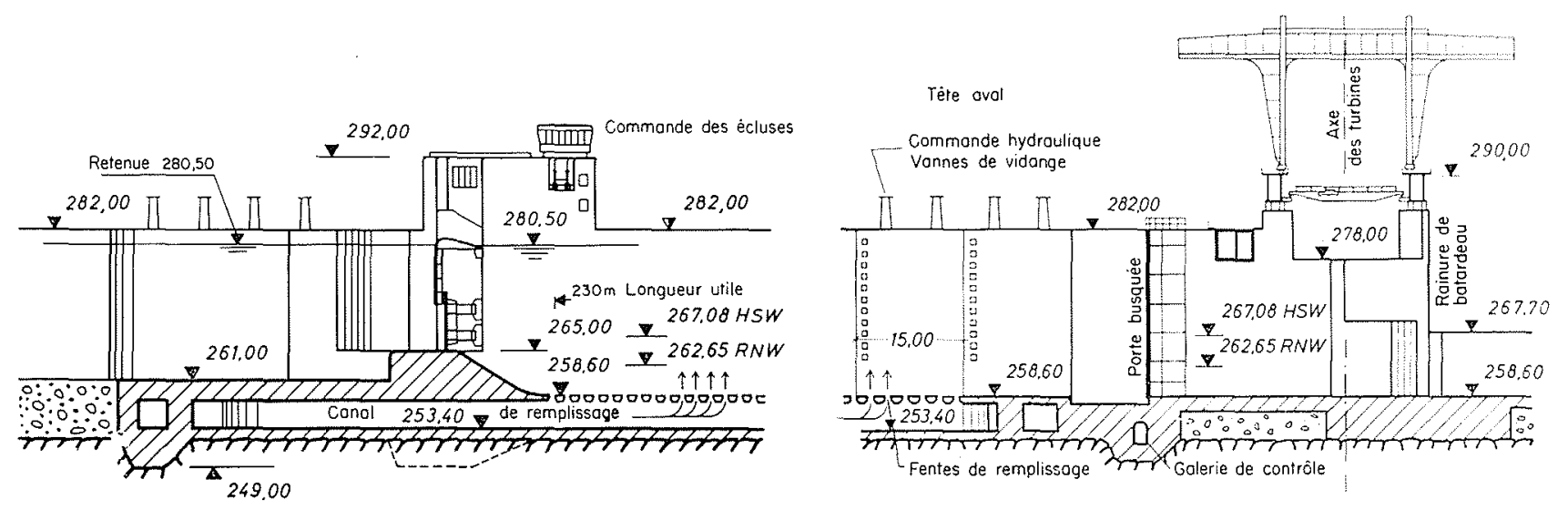

11/ Coupe en long d'une chambre d'écluse.

Längenschnitt durch eine Schleusenkammer.

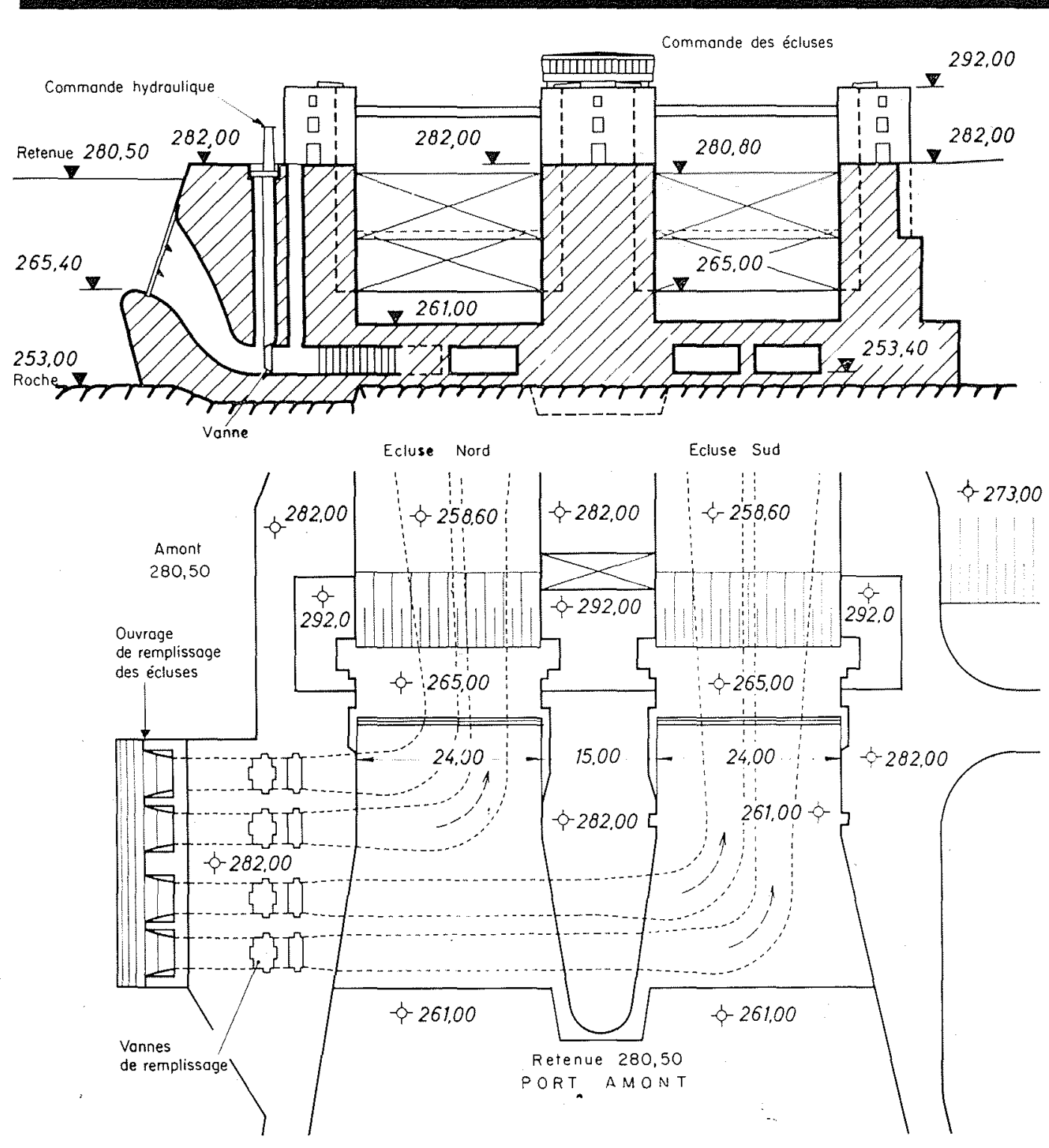

12/ Tête amont de l'écluse.

Schleusen-Oberhaupt. 


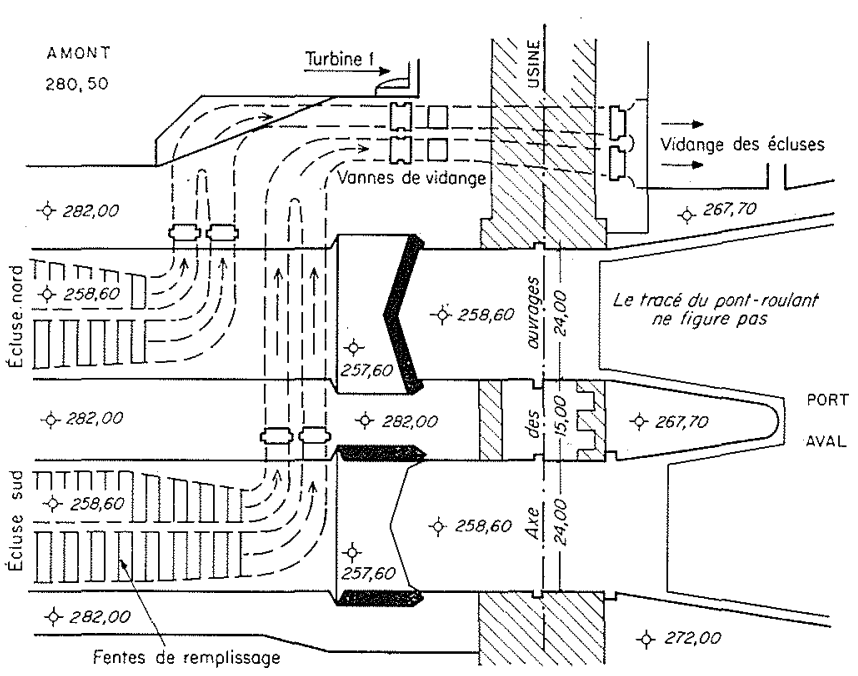

13/ Tête aval de l'écluse. Schleusen-Unterhaupt.

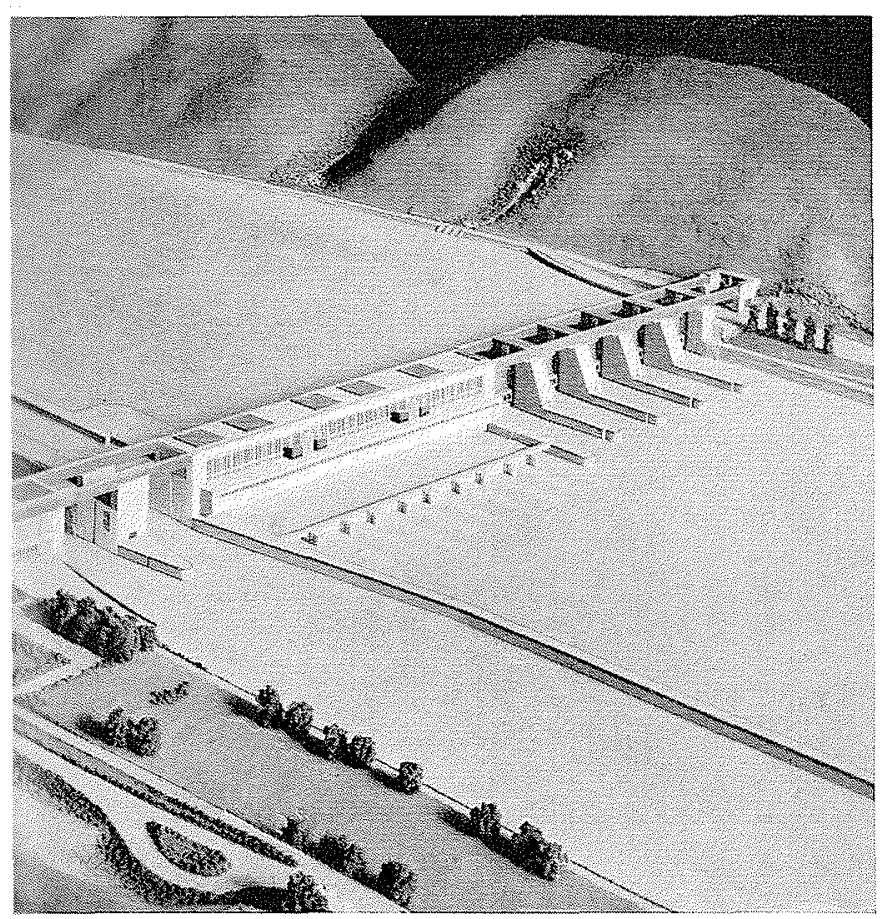

14/ Modèle de la centrale d'Aschach. Modell des Donaukraftwerkes Aschach.

des canaux sous le radier des sas. Au cours du remplissage, l'eau pénètre dans le sas par de nombreuses fentes prévues dans le radier, et y est uniformément répartie. Lors de la vidange, l'eau s'écoule à travers ces fentes dans les canaux, et ensuite par l'ouvrage de vidange dans le fleuve. Grâce à ce procédé de remplissage el de vidange, il ne sera probablement plus nécessaire d'amarrer les bateaux, car le niveau dans le sas monte et descend de manière très tranquille et régulière. D'autre part, aucun courant ne se produisant dans les avant-ports, les trains de touage peuvent manœuvrer, entrer et sortir sans danger. Des études approfondies sur un modèle d'écluse à l'échelle de $1 / 25$ ont montré qu'il est possible de réaliser, en 12 à $13 \mathrm{mn}$, le remplissage et la vidange d'un sas. A la tête aval, la fermeture de l'écluse est assurée par des portes busquées, à la tête amont au moyen de portes levantes-plongeantes du genre des vamnes doubles à crochet (fig. 11). En cas de crue catastrophique, celte disposition permet également l'évacuation par les écluses d'une partie des eaux. Des essais sur modèle ont montré que les deux écluses à elles seules peuvent évacuer environ $28 \%$ des débits de crue.

Le hall de montage et le bâtiment de commande de l'écluse sont situés sur la rive droite, à còté de la tête aval de l'écluse. Le chemin roulant d'une grande grue portique, d'une capacité portante de $220 \mathrm{t}$, franchit l'ensemble des installations, c'està-dire qu'il domine les toits du bâtiment de montage et de l'usine, ainsi que les ponts d'écluses et le barrage. Le montage et le démontage des groupes s'effectuent à travers des lucarnes aménagées dans le plafond de l'usine. La grue portique sert également à la mise en place des batardeaux à l'amont du barrage et des turbines, et à l'aval des installations d'écluses, ainsi qu’à amener et enlever les transformateurs.

La figure 14 monlre un modèle de la centrale danubienne d'Aschach, la construction étant achevée.

Contrairement à la centrale d'Ybbs-Persenbeug, aucun pont-route n'est prévu pour la centrale d'Aschach. Il aurait été extrêmement difficile et coûteux de faire passer un tel pont par-dessus les écluses, la centrale et le barrage, et d'encastrer les abords du pont dans les pentes raides des berges. Cependant, en collaboration avec l'Administraton de la Haute Autriche et l'Administration des Routes Fédérales, l’on construit actuellement, à la limite est de la ville d'Aschach, un pont-route moderne s'englobant librement et sans difficulté dans le réseau routier existant.

Comme il a déjà été dit, la retenue s'étend sur $40 \mathrm{~km}$ jusqu'en aval de la centrale de Jochenstein, située en amont. La retenue, haute d'environ $16 \mathrm{~m}$, affecte près de cent soixante propriétés qui doivent être, soit rachetées, soit reconstruites ou transformées. Elle affecte plus particulièrement les agglomérations d'Obermühl et d'Untermühl, qui seront rasées et reconstruites à une cote plus élevée. Dans la vallée de la rivière Grosse Mühl, la retenue s'étend jusqu'à la centrale de Partenstein (OKA) dont les turbines sont également à transformer. Les plaines bordant le fleuve seront exhaussées jusqu'à ce qu'elles dépassent d'au moins $1 \mathrm{~m}$ le niveau de la retenue. Elles seront de nouveau soumises ì la culture. Les talus des berges seront complétés et protégés contre les crues et le charriage do glaces.

En ce qui concerne la navigation, la hauteur de cette retenue apporte des avantages considérables en ce sens que les bancs de sable et les écueils se trouveront complètement submergés, et les vitesses d'écoulement fortement diminuées. D'autre part, la suppression de plusienrs coudes trop brusques permettra d'améliorer la visiblité et donnera une meilleure profondeur du chenal navigable, assurant ainsi, même à l'étiage, une navigation en double file.

Avant d'entreprendre la construction, l'on eut soin d'étudier, par de nombreux sondages, le niveatu et la composition du sol de fondation. Dans la zone de l'ouvrage principal, une semelle de granite presque plane s'étend d'un flanc à l'autre de la vallée, à une profondeur de près de $8 \mathrm{~m}$ sous le fond de la rivière. La surface de la roche a été aplanie par 
les crues noterienres; elle a été débarrassée de toulos les parties altérées et friables, et constitue donc, pour les ouvrages, un support sûr et idéal.

La seule difficulté réside en la profondeur à laquelle se trouve la semelle rocheuse sous le lit du lleuve, car elle oblige, pendant toute la durée des lravaux, à prévoir des hatardeaux et des digues de prolection anormalement hauts. Les résultats de caleuls et d'études très approfondis ont conduit à adopter et à réaliser, pour les enceintes, les types d'ouvrages représentés par la figure 15. Ces batardeaux, d'une hauteur de $19 \mathrm{~m}$, comptent parmi les plus importants ayant jamais été utilisés en Europe centrale.

La construction de la centrale d'Aschach s'effectue en trois tranches. La première tranche. (fig. 16) comporte la construction, à l'abri des batardeaux déjà décrits, de deux passes et de deux piles sur la rive gauche, et de l'écluse sud sur la rive droite. Simultanément l'on construit, au moyen de caissons à air comprimé, les fondations de la pile de séparation, prévue entre le barrage et la centrale. La navigation se déroule dans la section du fleuve située entre les caissons de la pile de séparation et le batardeau de la rive droite.

La deuxième phase de construction (fig. 17) comprend la construction de deux piles et des trois passes succédant aux deux passes déjà réalisées sur la rive gauche, par lesquelles s'effectue l'écoulement, ainsi que, sur la rive droite, la réalisation de la tête amont de l'écluse nord. Le montage de l'écluse sud sera également terminé au cours de cette phase de construction. Pendant la durée de ces travaux, la navigation s'effectuera dans la section encore libre du fleuve.

Au conrs de la troisième tranche de construction (fig. 18), après mise en service de toutes les passes, et après avoir dirigé la navigation sur l'écluse sud, l'on aménagera la fouille de la centrale au moyen de batardeaux allant de l'écluse jusqu'à la pile de séparation, et l'on commencera alors la construction des quatre blocs destinés à recevoir les groupes, ainsi que l'aménagement de l'écluse nord.

Actuellement, le barrage et l'écluse sud sont achevés, et depuis octobre 1961, la navigation s'effectue par l'écluse sud. Comme il est prévu dans la troisieme phase de construction (fig. 18), on travaille à la centrale et à l'écluse nord; l'eau du Danube passe déjà par le nouveau barrage. Si les travaux progressent normalement, on prévoit de créer, au cour's de l'été 1963, une retenue partielle jusqu'à la cote 276,50 , et de mettre en service le premier groupe. La retenue complète est prévue pour la fin de l'année 1963.

L'ourrage présente des dimensions exceptionnelles. Les parois des écluses ont une épaisseur de $15 \mathrm{~m}$ et une hauteur de $30 \mathrm{~m}$; la hauteur de la centrale, depuis le sol de fondation jusqu'à la corniche, est de $52 \mathrm{~m}$. Compte tenu de ces dimensions, les masses de construction à fabriquer sont, bien entendu, également extrêmement importantes. Pour l'ouvrage principal, la quantité de béton nécessaire est de l'ordre de 1,2 million de mètres cubes, dont environ $700000 \mathrm{~m}^{3}$ pour les écluses, et pour le barrage et la centrale, de près de $250000 \mathrm{~m}^{3}$, respectivement.

La quantité de roche qui, sur une profondeur de $15 \mathrm{~m}$, doit être extraite du gneiss le plus dur, sćlève à environ $270000 \mathrm{~m}^{3}$. Deux mille cing cents

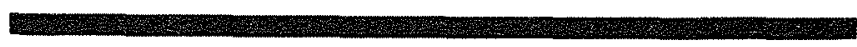

CELLULes Circulatres gATAROEAU
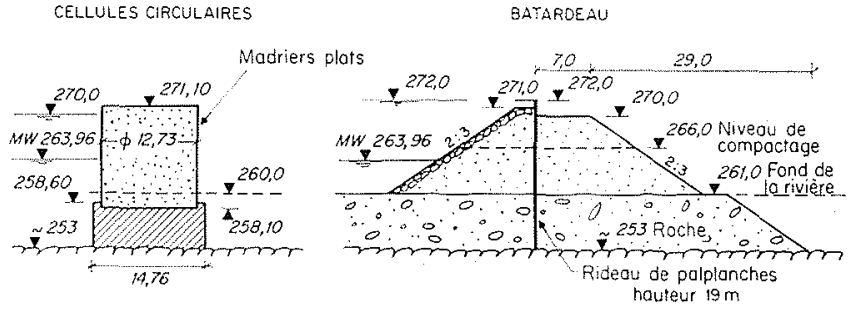

15/ Batardeaux des enceintes de chantiers.

Fangdämme der Baugrabenumschliessungen.

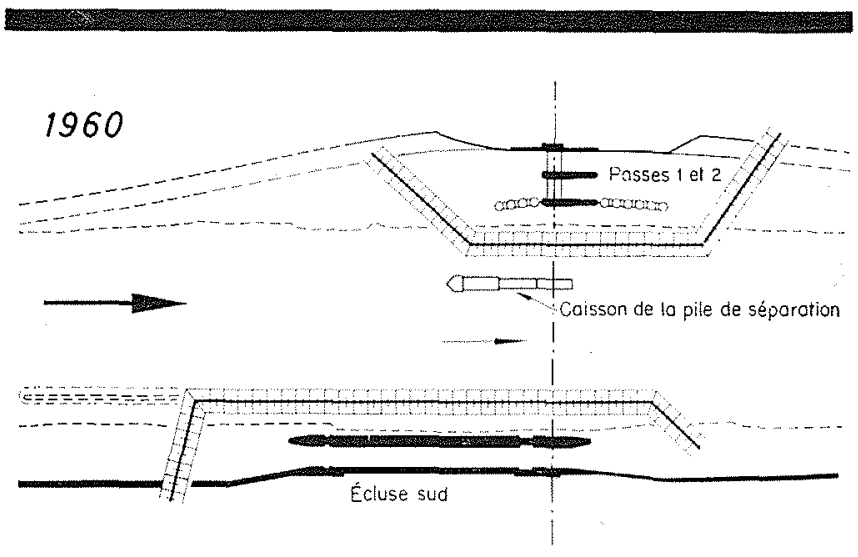

16/ Centrale d'Aschach. Phase 1 de la construction. Kraftwerl Aschach. Bauabschnitt 1.

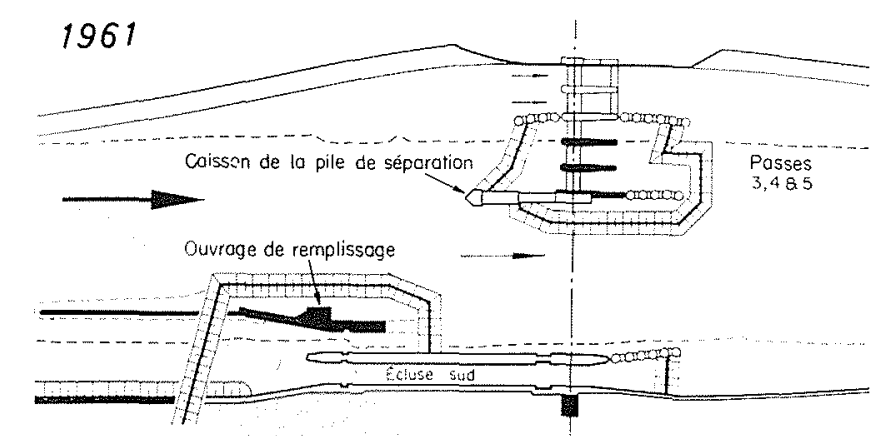

17/ Centrale d'Aschach, Phase 2 de la construction. Kraftwerk Aschach. Bauabschnitt 2.

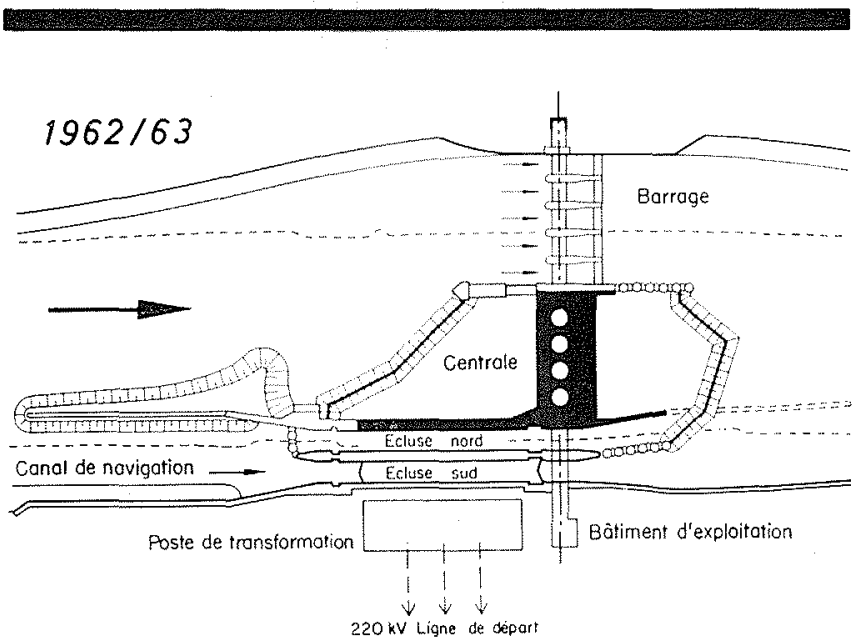

18/ Centrale d'Aschach. Phase 3 de la construction. Kraftwerk Aschach. Bauabschnitt 3. 
personnes participent à la construction, nombre certainement modeste si on le compare à l'importance des travaux à réaliser.

L'alimentation du chantier est assurée par des installations situées sur les deux rives, et équipées d'engins de production de gravier et de bétonnage. Sur la rive gauche, le transport s'effectue par camions; sur la rive droite, une voie ferrée normale, d'une longueur de $4,5 \mathrm{~km}$, relie le chantier à la gare d'Aschach. Cette voie de halage sert également au transport, jusqu'au chantier, des agrégats extraits et traités dans la plaine située à la limite sud de la ville d'Aschach.

L'ensemble des travaux ef prestations a fait l'objet d'un appel d'offres public et a été adjugé a des firmes ou groupes de travail. Un consortium formé de douze entreprises de construction est chargé de la construction de l'ouvrage principal, à l'emplacement de la chute. Compte tenu de l'importance des travaux et du délai de construction limité, les chantiers sont mécanisés dans une large mesure et équipés d'engins les plus modernes et les plus puissants. Pour l'exécution des travaux dans le fleuve, on dispose de nombreux bateaux ainsi que d'une grue flottante de $200 \mathrm{t}$. Un blondin, d'une longueur de $806 \mathrm{~m}$ et d'une capacité portante de $9 \mathrm{t}$, franchit la vallée.

En ce qui concerne la zone de retenue, les travaux sont en cours. Ils comprennent essentiellement : la construction d'environ $50 \mathrm{~km}$ de chemins de berges et d'intérêt local; la régularisation de près de 100 embouchures de rivières et de ruisseaux, comportant environ 50 pertuis et ponts; des exhaussements de berges de plus de 2 millions de mètres cubes, et la mise en place, pour la protection des berges, de près de $500000 \mathrm{~m}^{3}$ d'enrochements. S'y ajoutent encore d'importants travaux de transplantation et de reconstruction. La zone de retenue a été divisée en lots, et les travaux correspondants furent adjugés par la voie d'appels d'olfres publics.

Afin de pouvoir déterminer la portée et l'imporlance de l'effet de retenue sur les rives et les aggloinérations, il fut nécessaire d'effectuer, au préalable, sur tout ce troncon de fleuve long de $40 \mathrm{~km}$, un relevé de la zone riveraine, soit d'environ 1000 ha. En collaboration avec les propriétaires et les administrations, il fallut évaluer des centaines de maisons, d'objets et environ 2000 parcelles, et il fallut dixer, au cours de longues et pénibles discussions, le genre et le montant du dédommagement ou du remplacement.

Dans la zone de l'étage de retenue d'Aschach, près de 100 propriétés doivent être reconstruites ou modifiées, et adaptées à la future retenue. Quelques-unes de ces propriétés existent depuis plus d'un siecle, et il est souvent difficile de concevoir le projet d'une construction remplaçant l'ancienne et qui soit conforme au nouveau règlement sur les constructions et aux conceptions actuelles relatives à l'habitat. En même temps, toutes les reconstructions ou nouvelles constructions doivent étre conçues de telle manière qu'elles s'adaptent harmonieusement au caractère de la vallée du Danube. Par ailleurs, toutes les terres afrectées par la construction de la retenue seront cultivées de nouveau, et l'on utilisera, bien entendu, les végétaux propres à la vallée du Danube.

De nombreux puits de forage de la nappe ont été aménagés dans la zone de la retenue, et ils font l'objet d'un contrôle permanent, afin de pouvoir déterminer aussi bien le niveau actuel de la nappe souterraine que les prochaines variations qu’il présentera après la réalisation de la retenue. Une importante prospection géologique a été nécessaire pour connaitre la structure du sol de fondation, sa capacité portante, et sa compacité. A l'emplacement de l'ouvrage principal, environ cent sondages ont ainsi été effectués verticalement, sur la rive et dans le fleuve, et, sur une longueur de $300 \mathrm{~m}$, transversalement an fleuve, ainsi que, dans l'axe de l'ouvrage, un sondage horizontal dans la roche, à une grande profondeur sous le lit du fleuve.

Au lieu d'implantation du barrage, le niveau de retenue dépasse de $10 \mathrm{~m}$ le niveau de crue maximal observé jusqu'ici. Etant donné que les zones riveraines nouvellement aménagées dépasseront d'au moins $1 \mathrm{~m}$ le niveau de la retenue, et que les habitations seront reconstruites à une cote encore plus élevée, le danger de crue sera écarté à tout jamais pour la moitié inférieure de la retenue. De même, dans la moitié supérieure de la retenue, toutes les nouvelles constructions seront implantées de telle façon qu'elles ne puissent être atteintes par les crues; les deux berges seront soumises à une égalisation continue pour que, à l'avenir, les crues puissent s'écouler librement et sans être freinées par des obstacles. Autant qu'on en puisse juger, la suppression des bancs de sable, écueils, îlots et saillies de la berge devrait également exclure tout risque de formation de glace. D’ailleurs, des briseglace prévus à cet effet faciliteront la dérive des glaçons provenant d'une couche de glace éventuelle.

Un dépôt de matériaux semble peu probable, la majeure partie des matériaux apportés se déposant dans la retenue de la centrale de Jochenstein ou aux centrales sur l'Inn, en amont. Dans la partie inférieure de la retenue d'Aschach, un dépôt de matériaux fins (vase) est cependant possible, mais ne peut encore etre évalué.

Il est évident que l'établissement du projet et la construction d'une des plus importantes centrales en rivière d'Europe, exigent de tous les participants non seulement des connaissances, une expérience et des dons d'organisation de tonte première qualité, mais aussi de l'audace et une conscience professionnelle très poussée. En effet, la réalisation d'un ouvrage coûtant plus de 3 milliards de schillings, d'une part, réclame une construction à la fois très économique et rentable, et, d'autre part, impose aux spécialistes financiers et commerciaux le problème extrèmement difficile d'assurer -_ et dans les meilleures conditions possibles - son financement. Mais grâce à un labeur pénible et consciencieux, grâce aussi à la patience et à la persévérance, les plus grandes difficultés se trouvent à présent surmontées, et c'est plein de confiance que l'on peut attendre l'achèvement de la construction el le succes de celte entreprise.

\section{Bibliographie.}

H. Grenca. - Donauprobleme der Wasserkraftnutzung und Schiffahrt. Oesterr. Wasserwirtschaft, Wien, 1953, Heft 1.

o. Vas. - Zum Rahmenplan Donau. Oesterr. Wasservirtschaftsuerband, Wien, 1955.

O. VAs. - Geschichte und Tatsachen der Donaukraftnutzung in Oesterreich. Zeitschrift für Wasser-and Energiewirtschaft, Zürich, 1956 , Heft $5 / 6$. 


\section{F. MAKOVEC}

H. Bömmen. - Die Kraftwerksstufen der österreichischen Donau. Oesterreichische Ingenieurzeitschraift, 1958 , Heft 1 .

O. Hönn. - Das Donaukraftwerk Ybbs-Persenbeug. Deutsche Technik, 1938.

O. Vas. - Ueber das Unterwasserkraftwerk. Verlag Springer, Wien, 1947.

A. Grzrwiensin. - Flusskraftwerke und Stromwerke. Verlag Springer, Wien, 1948 .

A. Grzywienski. - Das Donaukraftwerk Ybbs-Persenbeng. Verlag Springer, Wien, 1949.

I. GrengG und H. LAUfFer. - Das Kraftwerk im Strom. Oesterr. Wasserwirtschaft, 1949, Heft 9/10.

H. Grassberger und F. Makovec. - Das Donaukraftwerk Ybbs-Persenbeug. Oesterr. Wasservirtschaft, Wien, 1951, Heft $\breve{b} / 6$.

R. Partu. - Ein neuer Vorschlag für Ybbs-Persenbeug. Oestert. Wasserwirtschaft, Wien, 1951, Heft 2.

Sonderheft. - Das Donaukraftwerk Ybbs-Persenbeug. Oe.Z.E. 1955 , Heft 9 , Wien.

Sonderdruck. - Strom aus Ybbs-Persenbeug. Verlag Bohmann, Wien-Heidelberg, 1957.
H. Böhmer und H, Mosbauer. - Die Donau - Oesterreichs Stromreservoirs. Die Stufe Ybbs-Persenbeug. Oe.Z.E., Wien, 1957, Heft 8.

H. BöHmer. - Das Donaukraftwerk Ybbs-Persenbeug. Wasser- und Energiewirtschaft, Zürich, 1959, Heft 5 .

Oesterreichische Donavkratrwerk A.G. - Das Donaukraftwerk Aschach. Schweizerische Banzeitang, 1959 , Heft 28.

F. Susax. - Das Donaukaftwerk Aschach und seine Maschinensätze. Maschinen Welt und Elektrotechnik, Wien, November 1960, Heft 12.

F. Makovec. - Das Donaukrattwerk Aschach. Banindustrie, Wien, 1960 , Heft 12 .

Sonderheft. - Das Donaukraftwerk Aschach. Oe.Z.E., Wien, 1962, Heft 5 .

H. Bömmer. - Das Donaukraftwerk Aschach. Die Wasserwirtschaft, 1962, Heft $8 / 9$

Sonderheft. - Das Donaukraftwerk Aschach. Energiewirtschaft, Wien, 1962, November.

H. Fucrs. - Die Donaustufe Jochenstein. Die Wasserwirtscha/t, 1953, Heft 11/12.

H. Böck. - Die Donaustauanlage Jrchenstein. Die Wasserwirtschaft, April 1957.

\section{Zusammenfassung \\ Der Ausbau der Wasserkräfte der österreichischen Domau. Das Donaukraftwerk Aschach Von F. Makovec *}

Die Donau entspringt im deutschen Schwarwald, durchfließt oder berührt auf ihrem $2848 \mathrm{~km}$ langen Weg 8 Staatsgebiete und mündet schließlich in das Schwarze Meer. Bereits im Altertum bildete die Donau die natürliche Nordgrenze des römischen Weltreiches und einen sehr wichichtigen Verkehrs - und Transportweg, wenngleich nur eine Kleinschiffahrt mit Ruderbooten und Flößen möglich war. Die Einführung der Dampfschiffahrt vor über 1 Jahrhundert brachte dann plötzlich eine grundlegende Aenderung. Der verwilderte Strom mit seinen Klippen, Sandbänken und Stromteilungen genügte nun nicht mehr den Anforderungen der Dampfschifffahrt. Alle Uferstaaten waren gezwungen, den gesamten Stromlauf bis zu seiner Mündung einer großzügigen Regulierung und Sicherung zu unterwerfen. Diese Arbeiten dienten gleichzeitig auch dem Hochwasserschutz der am Strom gelegenen Siedlungen und Städte. Dieses große und kostspielige Regulierungswerk war um die Jahrhundertwende größtenteils abgeschlossen.

Die stürmische industrielle Entwicklung am Ende des vorigen Jahrhunderts hatte einen immer größer wer. denden Bedarf an Energie im Gefolge, der anfänglich durch Dampfkraftanlagen gedeckt werden konnte. Immer mehr aber wurde der Blick der Energiefachleute auf die bisher nicht verwerteten Wasserkräfte der Flüsse und Ströme gelenkt. Aber erst die Entwicklung und Verbesserung der Wasserturbinen und Stromerzeuger und besonders die Vervollkommnung der Transformatoren und die Möglichkeit, die gewonnene elektrische Energie mit beliebiger Spannung über große Entfernungen zu leiten, gab dem Ausbau der Waserkräfte einen stürmischen Impuls.

Der ungewöhnlich große Energieinhalt der österreichischen Donau hat gleichfalls zu zahlreichen Wasserkraftprojekten Anlaß gegeben. Leider behandelten diese Projekte jeweils nur Teilabschnitte der Donau und nahmen keine Rücksicht auf die Möglichkeit eines Ausbaues der anschließenden Stromstrecken.

Auf Grund dieser Erfahrungen entwickelte Prof. Dr O. Vas den Grundsatz einer restlosen Ausnützung der gesamten österreichischen Donaustrecke. Auf seinen Vorschlag wurde 1946 ein besonderer «Donau-Ausschuß» gegründet, der im folgenden Jahr 1947 der Oesterreichischen Donaukraftwerke A. G. die Aufgabe übertrug, das Projekt einer lückenlosen Kraftwerkskette der österreichischen Donau auszuarbeiten.

Es ist selbstverständlich sehr schwierig, einen der größten Ströme Europas, der breite fruchtbare Ebenen und dicht besiedelte Gebiete durchfließt, in eine lückenlose Kraftwerkskette umzugestalten. Bei der Ausarbeitung dieses Projektes mufiten die vielfachen, einander oft widersprechenden Wünsche und Forderungen der verschiedenen Kreise der Volkswirtschaft und Kultur nach dem Grad ihrer Wichtigkeit und Nützlichkeit abgewogen und entsprechend berücksichtigt werden.

Das Projekt der Oesterreichischen Donaukraftwerke A. G. enthält eine lückenlose Kette von 13 österreichischen Donaukraftwerken. Im Westen schließt diese Kette an das im Jahre 1956 fertiggestellte deutsch-österreichische Gemeinschaftskraftwerk Jochenstein an. An der Ostgrenze soll die Kette durch ein tschechoslowakisch-österreichisches Gemeinschaftskraftwerk Wolfstal fortgesetzt werden.

Nach Fertigstellung der gesamten Kraftwerkskette steht Oesterreich aus den Wasserkräften der Donau eine jährliche Energiemenge von rund 14,5 Milliarden $\mathrm{kWh}$ zur Verfügung. Das erste Kraftwerk Ybbs-Persenbeng ist bereits seit 1958 fertig und liefert jährlich $1250 \mathrm{GWh}$. Das nächste und größte Flußkraftwerk Mitteleuropas in Aschach wird im folgenden Jahre 1963 in Betrieb genommen und liefert nach seiner Fertigstellung 1650 GWh in das österreichische Verbundnetz.

* Dr Techn. und Bandirelor des Oesterreichischen Donaukraftwerke A, $\mathrm{s}$ 